\title{
Exploring the Empowerment of Older Adult Creative Groups Using Maker Technology
}

\section{Olivia K. Richards}

College of Information Sciences and Technology

The Pennsylvania State University

University Park, Pennsylvania, USA

Proactive Health Informatics REU Site

School of Informatics and Computing

Indiana University

Bloomington, Indiana, USA

orichards@psu.edu
Permission to make digital or hard copies of part or all of this work for personal or classroom use is granted without fee provided that copies are not made or distributed for profit or commercial advantage and that copies bear this notice and the full citation on the first page. Copyrights for third-party components of this work must be honored. For all other uses, contact the owner/author(s). Copyright is held by the

author/owner(s).

CHI 2017, May 6-11, 2017, Denver, CO, USA.

ACM ISBN 978-1-4503-4656-6/17/05.

http://dx.doi.org/10.1145/3027063.3048425

\begin{abstract}
Human-computer interaction researchers actively engage in technological design to empower older adults to live independent, active lives; however, the number of researchers who allow older adults to participate in the design and development process varies greatly. We leveraged past participatory design techniques with older adults by cooperatively learning their craft and carefully enhancing their crafting practices using small electronics. In doing so, older adults will have an opportunity to enhance their craft artifacts to best fit their health needs. The purpose of this needs assessment, including 8 hours of observation and 2 hours of beta testing, is to understand how older adults might use maker technology in their crafts and hobbies to positively impact their health. By facilitating beta testing and observing local crafting groups of older adults, we identified crafting activities that older adults participate in and learned how to best tailor technology to their crafts.
\end{abstract}

\section{Author Keywords}

participatory design; older adults; maker technology; arduino; paper crafts

\section{ACM Classification Keywords}

H.5.m [Information interfaces and presentation]: Human computer interaction $(\mathrm{HCl})$ 


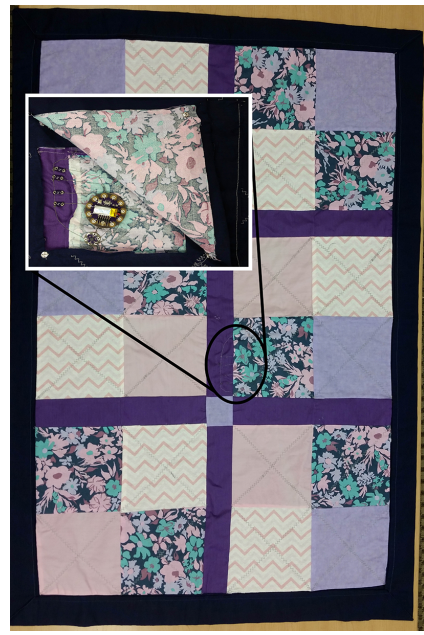

Figure 1: Research team's demonstration quilt, which included a LilyPad Arduino with conductive thread, LEDs, and an accelerometer. The cutout is the quilt's back flap hiding the electronics.

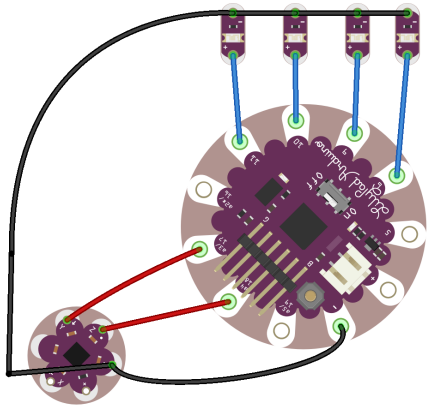

Figure 2: Fritzing Diagram of the Electronically Enhanced Quilt

\section{Background and Related Work}

Older adults possess a wealth of skills and life experiences that contribute to their post-retirement activities and can help to improve their lives and respective communities. Indeed, the expertise available will increase as baby boomers retire. Passionate crafting and hobbyist groups are growing in communities across the country. Although some researchers discuss the burdens presented by this generation, we encourage the research community to consider the skill sets of older adults and ways to empower them to tailor technology to their crafts rather than tailor their crafts to technology.

Researchers have found that stereotypes of older generations being unproductive and having an anti-technology mindset is inaccurate and makes their involvement in design activities unsuccessful [5]. Lee et al. [4] found that numerous older adults have a clear motivation for learning to satisfy their emotional and intellectual needs. Older adults have unique ideas that are simply not being considered [2]. A user-centric design approach can be more successful than the traditional patient-centric approach in technological development [2]. Technological gaps must be closed to avoid the vicious cycle of technology development which excludes older adults, and move toward a cycle which includes older adults in the development process [2]. In the past, older adults have assisted designers in tailoring their products to the needs of this population through participatory design workshops [5]. Our work, however, takes a step back from constraining goals and encourages flexibility in older adult creative technology.

Researchers successfully enhanced crafting practices with electronics in fabric [1] and paper [7], using specific components such as microcontrollers [6] like the Lilypad Arduino. Lee and Dey enhanced everyday objects [3] through home sensing while tracking both phone usage and medicine intake to improve the age-in-place process. With more data awareness, participants noticed the errors of habit, wanted to fix them, and became more willing to share these concerns with their care network, showing promise in effective health care measures. Even though researchers have worked with older adults in participatory design settings, there has not been a cohesive study that investigates how to empower older adults to integrate small electronics into their preferred crafting techniques and practices.

The purpose of this study is to understand how older adults might implement electronics into their crafts and learn how to interact with older adult crafters to encourage and empower them to track, remind, or monitor their health using small electronics. We identified the types of educational crafting activities that are important to include in the participatory design process, what health knowledge and skills are helpful to teach, and what practices are most beneficial for future work. We created exemplars and built on past participatory design techniques by studying the intersection between electronic engagement and health monitoring.

Our work provides the human-computer interaction community a better understanding of how to respectively integrate small electronics into the crafting lifestyle of older adults. This includes analyzing the types of devices that older adults would be interested in creating based on their craft. We suggest ways to introduce electronics into established older adult crafting communities.

Although researchers have done user-centered and expertcentered participatory design studies [2, 5], commonly noted challenges persist. To overcome insincere involvement of facilitators, future studies with older adults should be designed and approached by researchers maintaining focus and structure in meetings, representing and acting 


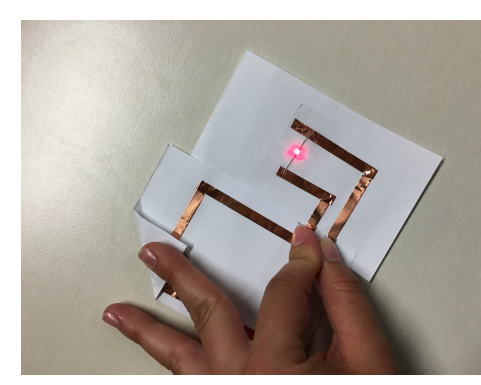

Figure 3: Simple two-piece paper circuit activity from the participatory design beta test on issues, envisioning intangible concepts, and designing for non-tasks [5]. Workshop and design facilitators should work with older adult participants while designing and employ a hands-off approach to give them the opportunity to contribute in whatever way they feel comfortable [2].

Other researchers have investigated what older adults might want to track related to their health [2], but they used participatory design methods without ample time for participant reflection. Participatory design methods are more successful in terms of older adults making a contribution when based on the endeavor to empower the older adult participants, instead of simply aiding them [8]. We instead focus on how to engage older adults in their established crafting groups.

Rogers et al. [8] facilitated creative workshops with older adults who interacted with MaKey MaKey toolkits. They noted how all groups between the ages of 60 and 80 surpassed expectations by mastering the technology with intense focus and diverse idea creation [8]. Our work enhances flexibility in the personalization of electronics within the realm of crafting and health-sensing. We have investigated the practices necessary to empower older adults to integrate their preferred crafting techniques/practices with maker technology.

\section{Methods}

Observation of Crafting Groups

Researchers visited two local crafting groups including Discardia, a public crafting event for all ages, and a local senio center lap quilting group. Observations of the individuals and selected domains were recorded by hand using temporal mapping. Notes were later transcribed electronically and qualitatively analyzed using an Affinity Diagram to detect commonalities and differences between the crafting events.
At Discardia, we analyzed typical behaviors of crafters, hobbyists, and facilitators. We learned how older adults shared, learned, and facilitated their crafting experiences. The opportunity to incorporate electronic components into crafting became evident.

\section{Participatory Design Beta Testing}

The researchers recruited colleagues for participation and developed a participatory design workshop to teach basic circuitry to older adult crafters. We created a process, through beta testing, in which participants would be encouraged to create their own simple paper circuit projects using copper tape, a 3V battery, and LED lights. The beta testing participants learned about inputs, outputs and their proper assembly. The researchers analyzed the process and answered relevant questions such as: What was and was not successful? Were any tasks difficult or challenging, and if so, which ones? How can the process improve to become more efficient? and What would you do different next time? Researchers facilitated the workshop, received feedback, and captured the qualitative data using artifacts, notes, photos, and recording methods.

\section{Prototype Exemplars}

The researchers mastered two crafts and created artifacts to serve as motivating examples for older adult participants in future participatory design workshops. The researchers produced a scrapbook with a light sensor to track generational usage of the scrapbook. Additionally, they learned the immensity of the quilting process while creating a quilt that integrated a health sensing component for mindful tracking The artifacts created by the researchers show the endless possibilities using LilyPad Arduino microcontrollers, as well as the inherent flexibility that comes with personalized creation to achieve goals through health sensing. 


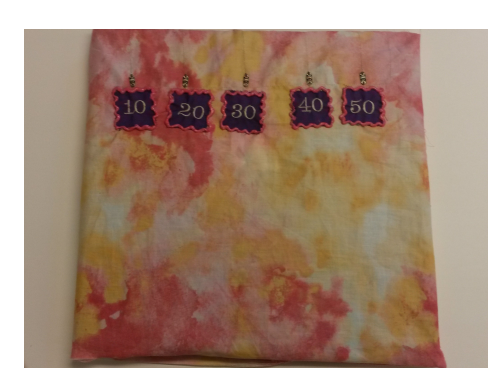

Figure 4: Electronically Enhanced Scrapbook

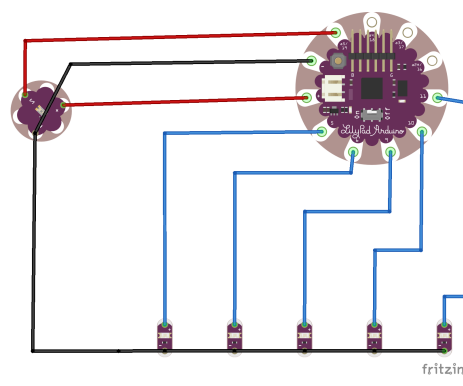

Figure 5: Fritzing Diagram of the Electronically Enhanced Scrapbook

\section{Electronically Enhanced Scrapbook}

The researchers composed a scrapbook (see Figure 4) to creatively hold memories of the first Proactive Health Informatics REU at Indiana University. In this short time frame, researchers crafted while learning how to program the Arduino LilyPad microcontrollers to integrate electronics into the craft.

The scrapbook had a light sensor that triggered the LilyPad to count how many times the book had been opened, and then display the amount on the cover of the book with LEDs. The LEDs, microcontroller, and light sensor were all sewn into a handmade fabric book cover that covered the scrapbook. The five LEDs were programmed using Arduino to display the total count of viewing occurrences with each diode representing ten occurrences. The purpose of electronic incorporation into this scrapbook was to display the number of people who have viewed and contributed to the scrapbook over generations.

\section{Electronically Enhanced Quilt}

The researchers mastered the quilting process in 5 weeks and continued to become proficient in programming the Arduino LilyPad microcontrollers for e-textile enhancement. The researchers created and programmed the quilt to monitor rest time in order to encourage mindfulness of stationary time and the need for daily activity. The LilyPad input pieces detect inactivity using an accelerometer and output pieces display the usage, lighting 1 LED for every thirty minutes of inactivity.

We calibrated the accelerometer to detect inactivity patterns. We verified the center as the location for the accelerometer through testing by placing the sensor in different places on a blanket. Researchers graphed the data from that beta test of blanket usage and recorded changes that were needed for an effective range.
In addition, the quilt can be used to encourage older adults' awareness of personal resting habits and mindfulness of needs for change. For example, if someone recently had surgery and has to rest for a certain amount of time each week, then they can be more conscious of the frequency of activity for post-operation healing. Or, if an older adult does not rest enough, the quilt can track rest and display sleep time.

\section{Results}

During the beta testing, observing, and crafting, researchers discovered how to respectfully interact with creative older adults, what to include in the process of facilitating future participatory design workshops, and what efforts are needed to craft and integrate electronics.

\section{Observation}

In a suburban college town, three researchers attended a local senior center's weekly lap quilting group meeting on a few occassions and learned how to best interact with older adult crafters who passionately quilt. The weekly meeting provided a common space to learn and work on projects, as well as a social community-building opportunity for the crafters. Immediately, there was a common urge amongst the quilters to "show-and-tell" their personal work and others, and share their personal crafting motivations, including self-benefit, personal profit, gifting, and charitable work.

Quilting took on a unique and different definition amongst crafters in this group. It was described as a lifestyle, a "sickness," and an "addiction," far beyond a simple hobby or craft. The quilters noted how quilts have distinct purposes, including resting, comfort and decoration. We quickly realized that the various differences present technological implications. For example, wall hangings may require ambient display style electronics, but not require durability, while 


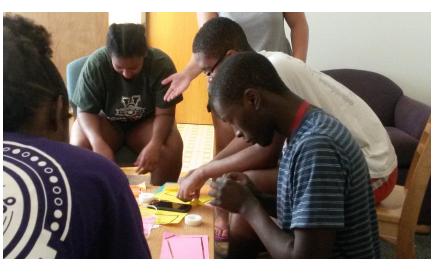

Figure 6: Research team's participatory design beta test wearable quilts for comfort could utilize wearable sensors and must have durable and secure electronic components. This experience profoundly impacted the way that we incorporated technology into the quilt and how we will communicate this to older adults.

The quilters technological experience encompassed the entire spectrum. But, with an introduction to our work, their openness to technology became more apparent and there was true interest in technological functionalities being tailored to their needs.

During the second lap quilting session, researchers reiterated previously unanswered questions and took note of relevant information for future $\mathrm{HCl}$ work. The quilters proudly held their projects in a show-and-tell fashion. There was frequent conversation regarding the fact that crafters should enjoy their own work. The group emphasized the importance of keeping tasks fun, relaxing and not too challenging. In future work, researchers should be mindful of this to provide a balance between tasks. The quilters reflected on the previous session and asked the observers questions about working with microcontrollers and e-textiles. For example, one quilter asked if one of their current projects could be made interactive by playing a tune when pressing different quilt squares, a possible task using a LilyPad buzzer. Another quilter conceptualized previous e-textile conversation and thought about turning wall quilts into ambient displays. This further emphasizes the openness amongst this creative community to incorporating technology into crafting practices.

In analyzing our observational sessions, we identified three major themes including group structure, group support, and artifact sharing, all of which help to explain the value in working with established older adult crafting groups.
Beta Test of Participatory Design Workshop

During the beta testing process, we learned the importance of participants being able to slowly follow along with the facilitator in a show-and-tell-like approach to ensure clarity. We also realized that presentation methods should vary in method including verbal, tangible, and electronic demonstration. While the planned tasks may have seemed simple to our tech-savvy participants, they may be foreign to a demographic unaccustomed to this domain. Facilitators should be aware of this gap of knowledge, keep a slow instructional pace and allow older adults of all skill levels to keep up, yet maintain a challenging balance. Participatory design workshops should be tailored to an environment of open discussion and collaboration.

Working with Creative Older Adults

To fully engage and teach older adults, methods of encouraging independence and creativity can be achieved by tailoring the participatory design environment to a wide range of skill levels and acting in the mere background until help is needed.

The exemplars produced can motivate experienced crafters to electronically enhance their work and provide a mechanism to disseminate the knowledge. The researchers began the projects without prior textile-based crafting skills and learned how much hard work and practice is needed to complete a project with proficiency. The mere task of incorporating electronics into crafts increased researchers' awareness of how to use components such as sensors and conductive thread in the most aesthetically pleasing fashion. Additionally, researchers gained respect for the extent to which the older adults genuinely enjoy their "lifestyle" of crafting.

If researchers produce a kit of small electronics for e-textile use, it should be scaffolded to avoid failure and ensure that 
participants enjoy the process. When designing with and for older adults, researchers and designers should tailor electronic integration to their crafts, and not the other way around.

\section{Acknowledgements}

We thank all Indiana University ProHealth 2016 REU members and mentors, Dr. Katie Siek and Ben Jelen of Indiana University, and Samantha Whitman of Arizona State University. Also, we thank Dr. Eun Kyoung Choe for mentorship at The Pennsylvania State University. This work is supported in part by the National Science Foundation Award IIS-1439241. Any opinions, findings, and conclusions or recommendations expressed in this material are those of the author(s) and do not necessarily reflect the views of the National Science Foundation.

\section{References}

[1] Leah Buechley and Michael Eisenberg. 2009. Fabric $\mathrm{PCBs}$, Electronic Sequins, and Socket Buttons: Techniques for e-Textile Craft. Personal Ubiquitous Comput. 13, 2 (Feb. 2009), 133-150. DOI : http://dx.doi.org/10.1007/s00779-007-0181-0

[2] Jennifer L. Davidson and Carlos Jensen. 2013. What Health Topics Older Adults Want to Track: A Participatory Design Study. In Proceedings of the 15th International ACM SIGACCESS Conference on Computers and Accessibility (ASSETS '13). ACM, New York, NY, USA, Article 26, 8 pages. DOI : http //dx.doi.org/10.1145/2513383.2513451

[3] Matthew L. Lee and Anind K. Dey. 2011. Reflecting on Pills and Phone Use: Supporting Awareness of Functional Abilities for Older Adults. In Proceedings of the SIGCHI Conference on Human Factors in Computing Systems (CHI '11). ACM, New York, NY, USA, 20952104. DOI : http://dx.doi.org/10.1145/1978942.1979247
[4] Young S. Lee, Shirley Chaysinh, Santosh Basapur, Crysta J. Metcalf, and Hiren Mandalia. 2012. Active Aging in Community Centers and ICT Design Implications. In Proceedings of the Designing Interactive Systems Conference (DIS '12). ACM, New York, NY, USA, 156-165. DOI : http://dx.doi.org/10.1145/2317956. 2317981

[5] Stephen Lindsay, Daniel Jackson, Guy Schofield, and Patrick Olivier. 2012. Engaging Older People Using Participatory Design. In Proceedings of the SIGCHI Conference on Human Factors in Computing Systems (CHI '12). ACM, New York, NY, USA, 1199-1208. DOI : http://dx.doi.org/10.1145/2207676.2208570

[6] David A. Mellis, Sam Jacoby, Leah Buechley, Hannah Perner-Wilson, and Jie Qi. 2013. Microcontrollers As Material: Crafting Circuits with Paper, Conductive Ink, Electronic Components, and an "Untoolkit". In Proceedings of the 7th International Conference on Tangible, Embedded and Embodied Interaction (TEI '13). ACM, New York, NY, USA, 83-90. DOI : http://dx.doi.org/10.1145/2460625.2460638

[7] Jie Qi and Leah Buechley. 2010. Electronic Popables Exploring Paper-based Computing Through an Interactive Pop-up Book. In Proceedings of the Fourth International Conference on Tangible, Embedded, and Embodied Interaction (TEI '10). ACM, New York, NY, USA, 121-128. DOI : http://dx.doi.org/10.1145/1709886. 1709909

[8] Yvonne Rogers, Jeni Paay, Margot Brereton, Kate L. Vaisutis, Gary Marsden, and Frank Vetere. 2014. Never Too Old: Engaging Retired People Inventing the Future with MaKey MaKey. In Proceedings of the 32Nd Annual ACM Conference on Human Factors in Computing Systems (CHI '14). ACM, New York, NY, USA, 3913-3922. DOI : http://dx.doi.org/10.1145/ 2556288.2557184 